\title{
Neurocognitive Assessment in Stage 5 Chronic Kidney Disease Patients on Various Treatment Modalities
}

\author{
Manoharan Renugasundari'Subramaniyam Velkumary ${ }^{1,}$, Sreejith Parameswaran ${ }^{2}$, Nivedita Nanda ${ }^{3}$
}

\section{Manoharan Renugasundari', Subramaniyam Velkumary ${ }^{1, *}$, Sreejith Parameswaran², Nivedita Nanda ${ }^{3}$}

\section{'Department of Physiology, Jawaharlal Institute of Post-graduate Medical Edu- cation and Research, Puducherry, INDIA. 2Department of Nephrology, Jawaharlal Institute of Post-graduate Medical Edu- cation and Research, Puducherry, INDIA. ${ }^{3}$ Department of Biochemistry, Jawaha- rlal Institute of Post-graduate Medical Education and Research, Puducherry, INDIA." \\ Correspondence \\ Dr. S. Velkumary \\ Department of Physiology, Jawaharlal Institute of Post-graduate Medical Education and Research, Puduch- erry-605006, INDIA. \\ Phone: +91-9442213592 \\ Email: velkumary@gmail.com \\ History \\ - Submission Date: 20-09-2018 \\ - Review completed: 27-11-2018; \\ - Accepted Date: 30-12-2018.}

DOI : 10.5530/ijcep.2019.6.1.6

\section{Copyright}

(c) 2019 Phcog.Net. This is an openaccess article distributed under the terms of the Creative Commons Attribution 4.0 International license.

\begin{abstract}
Background and Aim: The prevalence of Chronic Kidney Disease (CKD) is expected to rise among the various non-communicable diseases in India. CKD is associated with cognitive impairment. It requires one among the various treatments such as renal transplantation, Hemodialysis (HD) and Continuous Ambulatory Peritoneal Dialysis (CAPD). So cognitive deficit is known but the magnitude of improvement on different methods of treatment is not well established. Therefore, the present study is undertaken to determine the cognitive functions using MMSE score and paper and pencil neurocognitive test battery in stage 5 chronic kidney disease patients on various methods of treatment such as HD, CAPD and conservative management and compare it with controls. Methods: 54 medically stable stage 5 CKD patients (18 in each treatment group) and 18 healthy controls were recruited for the study. Mini Mental State Examination (MMSE) and Paper and pencil neurocognitive battery such as Trail Making Test A (TMT A), Trail Making Test B (TMT B), Letter cancellation test (LCT), Forward Digit Span (FDS) and Backward Digit Span (BDS) along with Body Mass Index (BMI) and biochemical parameters such as Urea, Creatinine, Glucose, Hemoglobin were noted from case sheets and eGFR calculated using CKD-EPI. Results: The mean MMSE scores, TMT A, TMT B, LCT, FDS and BDS were prolonged in all three treatment groups but found to be more prolonged in CKD patients on conservative management $(p<0.001, p=0.008$, $p=0.039, p=0.059, p=0.135$ and $p=0.038$ ) respectively. Conclusion: Cognitive functions are more affected in stage 5 CKD patients on conservative management compared to patients on dialysis.
\end{abstract}

Key words: Cognition, Chronic kidney disease, Hemodialysis, Continuous ambulatory peritoneal dialysis, Conservative management.

\section{INTRODUCTION}

There is an alarming increase in non-communicable diseases in India, among which the prevalence of Chronic Kidney Disease (CKD) is expected to rise. The overall prevalence of $\mathrm{CKD}$ is reported to be about $17.2 \%$ in Indian population. ${ }^{[1]}$

The term Chronic Kidney Disease (CKD) is defined as sustained kidney injury for more than 3 months resulting in a GFR less than $60 \mathrm{ml} / \mathrm{min} / 1.73 \mathrm{~m} \cdot{ }^{[2]}$

$\mathrm{CKD}$ is classified into 5 stages. Stage 1 is kidney damage with or without increased GFR [GFR $\geq 90$ $\left(\mathrm{ml} / \mathrm{min} / \mathrm{m}^{2}\right)$ ], stage 2 is kidney damage with mild decrease in GFR [GFR: $60-89\left(\mathrm{ml} / \mathrm{min} / \mathrm{m}^{2}\right)$ ], stage 3 is moderate decrease in GFR [GFR: $30-59(\mathrm{ml} / \mathrm{min} /$ $\left.\mathrm{m}^{2}\right)$ ], stage 4 is Severe decrease in GFR [GFR: 15-29 $\left(\mathrm{ml} / \mathrm{min} / \mathrm{m}^{2}\right)$ ] and stage 5 is kidney failure [GFR: $\left.<15\left(\mathrm{ml} / \mathrm{min} / \mathrm{m}^{2}\right)\right]$.

All stages of CKD are associated with cognitive deficit. CKD has various complications which includes anemia, hyperlipidemia, hypertension, mineral bone disorder, volume overload, electrolytes and acidbase disturbances, cardiovascular disease and some common neurological complications are cognitive

impairment, stroke, encephalopathy, peripheral and autonomic neuropathies. ${ }^{[3-5]}$

Among these complications, cognitive deterioration in CKD patients remains underestimated.

The most commonly affected cognitive domains observed in patients with advanced CKD were memory, attention and executive function. ${ }^{[6,7]}$ A study by Tsai CF et al. says that the cognitive domains such as attention, visual tracking and working memory were affected in women with moderate CKD. ${ }^{[8]}$

Cognition is assessed by various neuropsychological tests. There are various methods to assess the cognition which includes MMSE for global cognition, Wechsler Intelligence Scale for Children and adult (WISC) to assess the general ability and intelligence, Paced Auditory Serial Addition Test (PASAT) to assess the speed processing, Digit span in WISC for attention and concentration, Wechsler memory scale to assess the memory and Boston Diagnostic Aphasia examination to assess the language ${ }^{[9]}$ and various paper and pencil neurocognitive tests. In this study, MMSE and Paper and pencil neurocognitive tests such as TMT A, TMT B, LCT, FDS, BDS were used.

Cite this article: Renugasundari M, Velkumary S, Parameswaran S, Nanda N. Neurocognitive Assessment in Stage 5 Chronic Kidney Disease Patients on Various Treatment Modalities. Int J Clin Exp Physiol. 2019;6(1):18-23. 
CKD, if untreated leads to ESRD which necessitates renal transplantation, Hemodialysis (HD) and Continuous Ambulatory Peritoneal Dialysis (CAPD). Kidney transplantation may even reverse some of cognitive deficits detected in the dialysis period, but the proportion of patients undergoing renal transplant for CKD is less due to many reasons, therefore they are mainly treated by dialysis. With regular treatment the cognitive function is expected to improve in CKD. But among the above listed treatment modalities, treatment that will improve the neurocognitive performance are poorly documented.

So cognitive deficit in CKD is known, but the magnitude of improvement on different methods of treatment is not well established and there is paucity of scientific data on impact of various modalities of treatment (such as CAPD, HD and conservative management for stage 5 CKD) on neurocognitive functions. Therefore, the present study is undertaken to bring out the appropriate treatment for ESRD, which would prevent the development of cognitive abnormalities in CKD patients.

\section{MATERIALS AND METHODS}

This is a cross-sectional study conducted in the Department of Physiology in collaboration with Department of Nephrology, JIPMER, Puducherry. The study was approved by JIPMER PG research monitoring committee (PGRMC) and Institute Ethics Committee (IEC) for human studies.

\section{Participants}

Medically stable 56 stage $5 \mathrm{CKD}$ patients were recruited for study who are on different modalities of treatment along with 18 healthy volunteers participated as control group. Staging of CKD was done based on the criteria given by Kidney Disease Outcome Quality Initiative (KDOQI) Foundation. Medically stable stage 5 CKD patients with eGFR $<8$ $\mathrm{ml} / \mathrm{min} / 1.73 \mathrm{~m}^{2}$ under different modalities of treatment such as $\mathrm{HD}$, CAPD and conservative management were recruited.

Group I ( $n=18$ )-Those undergoing hemodialysis twice a week for $\geq 3$ months duration.

Group II $(n=18)$-Those undergoing continuous ambulatory peritoneal dialysis for $\geq 3$ months duration.

Group III ( $n=18$ )-Those undergoing Conservative management $\geq 3$ months duration.

Group IV ( $n=18)$-Apparently healthy subjects of both gender.

\section{Inclusion Criteria}

1. Medically stable stage $5 \mathrm{CKD}$ patients with $\mathrm{eGFR}<8 \mathrm{ml} / \mathrm{min} / 1.73 \mathrm{~m}^{2}$ under different modalities of treatment such as HD, CAPD and conservative management for $\geq 3$ months. (CASES)

2. Apparently healthy individuals with normal creatinine level were included in the study. (CONTROLS)

3. Age between 18 and 60 years of either gender. (CASES and CONTROLS)

4. Formal education at least upto $6^{\text {th }}$ standard. (CASES and CONTROLS)

\section{Exclusion Criteria}

1. History of cerebrovascular disease, thyroid disease, severe anemia $\leq$ $8 \mathrm{gm} \%$, liver disease, respiratory failure.

2. Patients who are not on regular dialysis or medication.

All haemodialysis patients were asked to come, on the next day of dialysis for recording the study parameters. Informed consent was obtained from the participants before enrolling to the study.

\section{Sociodemographic and Biochemical Parameters}

All patient-related information such as age, gender, values of urea, creatinine, serum glucose and haemoglobin were noted from case sheet for CKD patients and eGFR was calculated with creatinine value using CKD-EPI.

Height and weight of the subjects were assessed.

Weight was assessed using weighing machine and height was assessed using stadiometer.

BMI was calculated using Quetlet's index

Quetlet's index = weight / [height ${ }^{2}$

Where, Weight was given in kilograms.

Height in centimeters

\section{Neurocognitive Tests}

\section{Mini Mental State Examination}

This is a valid and standardized tool to assess the global cognitive function. This assesses mainly five domains. They are orientation, attention, immediate and short term recall, language and visuo-spatial functioning. ${ }^{[10-13]}$ MMSE involved 11 questions. This test takes about $5-10 \mathrm{~min}$ to administer. The score range of this test is from 0 to 30 .

Scores $<24$ suggest the manifestation of decline in which,

\section{3-21 represents mild decline}

20-11 represents moderate decline

$<10$ represents sever decline $\mathrm{e}^{[14]}$

\section{Neurocognitive Test Battery}

Paper and pencil neurocognitive test which includes Trail Making Test A and B (TMT A and TMT B), Forward (FDS) and Backward Digit Span (BDS) and Letter Cancellation Test (LCT) was assessed.

\section{Trail Making Test A and B (TMT A and TMT B)}

It has been extensively used in the neuropsychological research for the assessment of psychomotor speed, complex attention and executive functions. It consists of two parts: TMT A and TMT B. TMT A assesses concentration, attention, visual scanning and tracking. The subject is instructed to draw lines connecting 25 consecutive circles. The score is the time (in sec) taken by the subject to complete the task. TMT B assesses attention, concentration, psychomotor speed, executive functions and set shifting. ${ }^{[15-18]}$ In this test the subject was instructed to connect 25 numbered and lettered circles by alternating between the two sequences. The score was the total time taken (in sec) by the subject to complete the task.

\section{Letter Cancellation Test}

LCT is commonly used to assess the psychomotor speed, visual scanning and sustained attention to identify the target letter in an array. ${ }^{[19]}$ In this test, the subjected was presented with 52 characters (English alphabets) in six rows and was instructed to strike out the randomly placed letter ' $\mathrm{H}$.' The score was the time taken by the subject to cancel out all the target letters in an array. And in addition, the number of different errors (omissions and commissions) done by the subject were also counted. Is the subject misses the target letters without striking, it is counted as omissions. And if the subject strikes out the non-target letters, it is counted as commissions.

\section{Digit Span}

It has two parts: Forward Digit Span (FDS) and Backward Digit Span (BDS). FDS assesses verbal attention and concentration. In this test, the subject was asked to repeat back the numbers in the same order that the 
examiner reads it. And the maximum number of digits that the subject was able to recite was given as a score. Backward digit span not only evaluates the aural attention and retaining capacity in short term but also the capability to manipulate the information in the verbal working memory. ${ }^{[20-22]}$ The subject was instructed to repeat the numbers in the reverse order. The maximum number of digits that the subject could recite in the reverse order was noted and given as a score.

\section{Statistical Analysis of Data}

Analysis was carried out using the Statistical Package of the Social Sciences (SPSS) version 20.0. Normality testing was done by One-Sample Kolmogorov-Smirnov Test. The parameters which passed through Gaussian distribution were expressed as mean with standard deviation. The parameters which did not pass through Gaussian distribution were expressed as median with interquartile range. In this study, anthropometric measurements such as age, height, weight, BMI and biochemical parameters such as urea, creatinine, eGFR, glucose, haemoglobin and neurocognitive test such as TMT A, TMT B, LCT, FDS, BDS passed Gaussian distribution. Therefore, it is expressed in mean with standard deviation. Whereas MMSE did not pass through Gaussian distribution, therefore it is expressed as median with interquartile range. One-way Analysis of Variance (ANOVA) was used to compare all the four groups and Post-Hoc test was done by Bonferroni to know the exact significance for parameters which followed normal distribution. Kruskal-wallis test was done to compare among all the four groups and Post-Hoc test was done by Tukey's test to know the exact significance for parameters which did not follow normal distribution. The difference was considered statistically significant if probability of chance was less than $0.05(p<0.05)$.

\section{RESULTS}

\section{Anthropometric Parameters}

Table 1 shows the comparison of anthropometric parameters between the control group and three treatment groups [CKD patients on (HD), (CAPD) and conservative management].

By design, there were no significant differences in age $(p=0.418)$ and height $(p=0.140)$ between various groups. There was a significant difference observed in the mean body weight between control group and HD patients $(p=0.028)$, between control group and CAPD patients $(p=0.014)$ and between control group and CKD patients on conservative management $(p<0.001)$. We also got significant difference in BMI among all the four groups $(p=0.002)$. In which significant difference was observed in BMI between control group and HD patients $(p=0.021)$, between control group and CAPD patients $(p=0.021)$ and between control group and CKD patients on conservative management ( $p=0.003)$.

\section{Neurocognitive Parameters}

Table 2 shows the comparsion of Neurocognitive parameters between healthy controls and reThere was a significant difference observed in LCT Omissions between all the four groups $(p<0.001)$. Post-Hoc test showed LCT (omissions) is significantly higher in CKD patients on conservative management $(p<0.001)$ than the healthy control. There is also significant increase in LCT omissions in CAPD patients $(p=0.02)$ than the control group. There was no significant difference observed in LCT score between the four groups ( $p=0.059$ ). LCT (Commissions) was not showed in the result as none of our subjects striked the non-target letters. One-way ANOVA showed a significant difference among all the four groups in TMT A $(p=0.008)$ and TMT B $(p=0.039)$. Post-hoc was done to know the exact significance. It revealed that significant difference was observed only between control group and CKD patients on conservative management in TMT A $(p<0.01)$ and TMT B $(p<0.05)$.
FDS was found to be decreased in all the three treatment groups when compared to the control group. One-way ANOVA showed that there is no statistical significant difference observed in FDS $(p=0.135)$. BDS showed a significant difference among all the four groups $(p=0.038)$ in which significant difference exist only between control group and CKD patients on conservative management $(p<0.05)$

\section{Mini Mental State Examination}

Table 3 shows the comparison of MMSE between the healthy controls and the treatment groups. MMSE was found to be decreased in all the three treatment groups when compared to the control group. It showed that there was a significant difference exist in MMSE among all the four groups $(p<0.001)$. In which significant difference was observed between control group and CKD patients on conservative management $(p<0.001)$, between control group and HD patients $(p<0.05)$ and between CAPD group and CKD patients on conservative management $(p<0.05)$.

\section{Biochemical Parameters}

Table 4 shows the comparison of biochemical parameters between control and all the three treatment groups. Serum urea was increased in all the three treatment groups than the control group. One-way ANOVA revealed that there was a significant difference in urea among all the four groups $(p<0.001)$. Post-hoc test showed that there was a significant difference in urea between HD group and control group $(p<0.001)$, between CAPD group and control group $(p<0.001)$, between control group and CKD patients on conservative management $(p<0.001)$, between HD group and patients on conservative management $(\mathrm{p}<0.001)$, between CAPD and CKD patients managed conservatively $(p<0.01)$.

Serum creatinine was increased in all the three treatment groups than the control group. One-way ANOVA test showed a significant difference in urea among all the four groups $(p<0.001)$. Post-hoc test showed that there was a significant difference in creatinine between HD group and control group $(p<0.001)$, between CAPD group and control group

\section{Table 1: Comparison of anthropometric parameters between control group and treatment groups.}

\begin{tabular}{|c|c|c|c|c|c|}
\hline 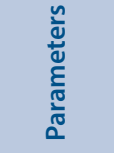 & $\begin{array}{l}\text { Control } \\
\text { Group } \\
(n=18)\end{array}$ & $\begin{array}{c}\text { HD } \\
(n=18)\end{array}$ & $\begin{array}{l}\text { CAPD } \\
(n=18)\end{array}$ & $\begin{array}{l}\text { Conservative } \\
\text { management } \\
\qquad(n=18)\end{array}$ & $\begin{array}{c}P \\
\text { value }\end{array}$ \\
\hline $\begin{array}{c}\text { Age } \\
\text { (years) }\end{array}$ & $39.50 \pm 10.55$ & $40.28 \pm 10.27$ & $42.61 \pm 10.69$ & $44.83 \pm 10.31$ & 0.418 \\
\hline $\begin{array}{c}\text { Height } \\
\text { (cms) }\end{array}$ & $160.94 \pm 5.86$ & $159.28 \pm 9.53$ & $158.33 \pm 8.18$ & $154.50 \pm 9.06$ & 0.140 \\
\hline $\begin{array}{c}\text { Weight } \\
\text { (Kgs) }\end{array}$ & $65.44 \pm 5.136$ & $56.23 \pm 9.48^{\star}$ & $55.47 \pm 11.47^{*}$ & $51.61 \pm 10.47^{\star * *}$ & $<0.001$ \\
\hline $\begin{array}{c}\text { BMI } \\
(\mathrm{Kg} / \mathrm{m} 2)\end{array}$ & $25.25 \pm 2.45$ & $22.05 \pm 3.36^{*}$ & $22.06 \pm 3.40^{*}$ & $21.44 \pm 3.31^{\star *}$ & 0.002 \\
\hline
\end{tabular}

Values expressed as Mean \pm SD. One-way ANOVA was done to compare the groups. Post Hoc test (Bonferroni test) was done to know the exact significance. The $p$ value $<0.05$ was considered to be statistically significant.

HD - Hemodialysis; CAPD- Continuous Ambulatory Peritoneal Dialysis; BMI Body Mass Index

* symbol shows comparison with control group

${ }^{\star} P<0.05 ;{ }^{* *} P<0.01$; ${ }^{* *} P<0.001$ 
Table 2: Comparison of cognitive parameters between control group and treatment groups.

\begin{tabular}{|c|c|c|c|c|c|}
\hline Parameters & $\begin{array}{l}\text { Control Group } \\
\qquad(n=18)\end{array}$ & $\begin{array}{c}\text { HD } \\
(n=18)\end{array}$ & $\begin{array}{l}\text { CAPD } \\
(n=18)\end{array}$ & $\begin{array}{l}\text { Conservative } \\
\text { management } \\
\qquad(n=18)\end{array}$ & $P$ value \\
\hline LCT (Omission) & $0.11 \pm 0.323$ & $1.28 \pm 2.539$ & $3 \pm 3.662^{\star *}$ & $3.28 \pm 1.809^{\star * \star}$ & $<0.001$ \\
\hline LCT (score) (sec) & $132.39 \pm 52.318$ & $158.33 \pm 70.517$ & $147.72 \pm 50.280$ & $185.28 \pm 58.825$ & 0.059 \\
\hline TMT A (sec) & $50.17 \pm 23.878$ & $68.44 \pm 48.097$ & $60 \pm 28.076$ & $98.11 \pm 58.938^{\star *}$ & 0.008 \\
\hline TMT B (sec) & $109.72 \pm 47.613$ & $134.11 \pm 75.692$ & $131.11 \pm 53.244$ & $172.72 \pm 77.269^{*}$ & 0.039 \\
\hline FDS & $5.78 \pm 0.943$ & $5.33 \pm 1.328$ & $5.28 \pm 1.227$ & $4.89 \pm 0.900$ & 0.135 \\
\hline BDS & $4.39 \pm 1.037$ & $4.17 \pm 1.098$ & $3.72 \pm 1.179$ & $3.39 \pm 1.092^{*}$ & 0.038 \\
\hline
\end{tabular}

Values expressed as Mean \pm SD. One-way ANOVA was done to compare the groups. Post Hoc test (Bonferroni test) was done to know the exact significance.

The $\mathrm{p}$ value $<0.05$ was considered to be statistically significant

* symbol shows comparison with control group

HD - Hemodialysis; CAPD- Continuous Ambulatory Peritoneal Dialysis; LCT - Letter Cancellation Test; TMT A - Trail Making Test A; TMT B - Trail Making Test B; FDS - Forward Digit Span;

BDS - Backward Digit Span

${ }^{*}(P<0.05) ;{ }^{* *}(P<0.01) ; * * *(P<0.001)$

Table 3: Comparison of MMSE between control group and treatment groups.

\begin{tabular}{cccccc} 
Parameters & $\begin{array}{c}\text { Control Group } \\
(\boldsymbol{n}=\mathbf{1 8})\end{array}$ & $\begin{array}{c}\text { HD } \\
(\boldsymbol{n}=\mathbf{1 8})\end{array}$ & $\begin{array}{c}\text { CAPD } \\
(\boldsymbol{n}=\mathbf{1 8})\end{array}$ & $\begin{array}{c}\text { Conservative } \\
\text { management } \\
(\boldsymbol{n}=\mathbf{1 8})\end{array}$ & $\begin{array}{c}\boldsymbol{P} \text { value } \\
\text { MMSE }\end{array}$ \\
\hline \multirow{2}{*}{$30.00(30.00-30.00)$} & $28.50(25.00-30.00)^{*}$ & $28.00(27.00-29.00)$ & $24.50(22.00-26.00)^{* * *},{ }^{+}$ & $<0.001$
\end{tabular}

HD - Hemodialysis; CAPD- Continuous Ambulatory Peritoneal Dialysis

Values expressed as Median (Interquartile range). The $p$ value $<0.05$ was considered to be statistically significant. Kruskal-wallis was done to compare the groups. Post Hoc test (Tukey's Hinges test) was done to know the exact significance.

${ }^{*}$ symbol shows comparison with control group; ${ }^{\dagger}$ symbol shows comparison with CAPD.

${ }^{*}(P<0.05) ;{ }^{* *}(P<0.01) ;{ }^{* * *}(P<0.001){ }^{\dagger}(P<0.05) ;{ }^{\dagger \dagger}(P<0.0) 1 ;^{\dagger+\dagger}(P<0.001)$.

Table 4: Comparison of biochemical parameters between control group and treatment groups.

\begin{tabular}{|c|c|c|c|c|c|}
\hline Parameters & $\begin{array}{l}\text { Control Group } \\
\qquad(n=18)\end{array}$ & $\begin{array}{c}\text { HD } \\
(n=18)\end{array}$ & $\begin{array}{l}\text { CAPD } \\
(n=18)\end{array}$ & $\begin{array}{l}\text { Conservative } \\
\text { management } \\
\qquad(n=18)\end{array}$ & $P$ value \\
\hline Urea $(\mathrm{mg} / \mathrm{dl})$ & $27.44 \pm 5.78$ & $74.89 \pm 23.59^{* * *}$ & $72.39 \pm 28.56^{* * *}$ & $107.94 \pm 32.99^{\# \# *,+t, * * *}$ & $<0.001$ \\
\hline Creatinine $(\mathrm{mg} / \mathrm{dl})$ & $0.94 \pm 0.137$ & $7.88 \pm 1.676^{* * *}$ & $9.56 \pm 3.029^{* * *}$ & $7.11 \pm 1.71^{\dagger+, * * *}$ & $<0.001$ \\
\hline eGFR & $91.33 \pm 18.53$ & $6.88 \pm 1.07^{* * *}$ & $5.97 \pm 2.637^{*+*}$ & $7.56 \pm 1.338^{* * x+}$ & $<0.001$ \\
\hline Glucose (mg/dl) & $96.67 \pm 14.72$ & $96.06 \pm 22.14$ & $101.22 \pm 30.32$ & $84.83 \pm 18.43$ & 0.160 \\
\hline $\mathrm{Hb}(\mathrm{gm} \%)$ & $11.56 \pm 1.464$ & $8.89 \pm 1.323^{* \star *}$ & $9.50 \pm 1.200^{* * *}$ & $9.56 \pm 1.097^{*+* x}$ & $<0.001$ \\
\hline
\end{tabular}

HD - Hemodialysis; CAPD- Continuous Ambulatory Peritoneal Dialysis

Values expressed as Mean $\pm \mathrm{SD}$. The $p$ value $<0.05$ was considered to be statistically significant. One-way ANOVA was done to compare the groups. Post Hoc test (Bonferroni test) was done to know the exact significance.

* symbol shows comparison with control group; ${ }^{*}$ symbol shows comparison with HD group

${ }^{\dagger}$ symbol shows comparison with CAPD.

${ }^{*}(P<0.05) ;{ }^{* *}(P<0.01) ;{ }^{* * *}(P<0.001) ;{ }^{*}(P<0.05) ;{ }^{* \#}(P<0.01) ;{ }^{* \# *}(P<0.001) ;{ }^{\dagger}(P<0.05) ;{ }^{\dagger \dagger}(P<0.01) ;{ }^{\dagger \dagger}(P<0.001)$

$(p<0.001)$, between control group and CKD patients on conservative management $(p<0.001)$.

Since significance was observed in creatinine, we also got statistical significant difference in eGFR among all the four groups $(p<0.001)$. Posthoc test was done to know the exact significance. In which significant difference was observed in eGFR between HD group and control group $(p<0.001)$, between CAPD group and control group $(p<0.001)$, between control group and CKD patients on conservative management $(p<0.001)$. There was no statistical significance difference observed in glucose among all the four groups $(p=0.160)$.

Hemoglobin was decreased in all the three treatment groups than the control group. One-way ANOVA test showed a significant difference in hemoglobin among all the four groups $(p<0.001)$. Post-hoc test showed 
that there was a significant difference observed in hemoglobin between HD group and control group $(p<0.001)$, between CAPD group and control group $(p<0.001)$, between control group and CKD patients on conservative management $(p<0.001)$.

\section{DISCUSSION}

In this study, we have assessed the cognition using MMSE and neurocognitive test battery along with biochemical and anthropometric parameters. The parameters assessed were paper and pencil neurocognitive tests such as Trail Making Test A (TMT A), Trail Making Test B (TMT B), Forward Digit Span (FDS), Backward Digit Span (BDS), Letter Cancellation Test (LCT) and Mini Mental State Examination (MMSE).

The results revealed that on general assessment of cognition by MMSE and neurocognitive test battery, the patients had lower scores of cognitive functions in all the three treatment groups in comparison to controls.

In MMSE, there was a significant difference observed in HD and CAPD and the values are found to be closer to control group.

Drew DA et al. ${ }^{[23]}$ Conducted a study in hemodialysis patients and found that there were decline in performance over a period of time in Trails A and $\mathrm{B}$ which may be due to underlying cerebrovascular neuropathology in HD patients.

The results revealed that the patients in CAPD treatment had better executive functions in comparison to other treatment groups. The data showed that the patients in CAPD treatment had better attention in comparison to other treatment groups as was revealed by the time taken to complete the letter cancellation tasks. A study conducted by Dixit A et al. ${ }^{[24]}$ who compared the cognitive functions between hemodialysis patients and healthy controls and on analysis, it was revealed that the controls took significantly shorter time than pre-dialysis state. However, after dialysis there was no significant difference observed in the performance of controls and dialysis patients. And also, controls had significantly less number of omissions when compared to dialysis.

Another study by Conde SAL et al. ${ }^{[14]}$ conducted on CKD patients who are on hemodialysis and peritoneal dialysis observed that forward digit span was worse among patients undergoing hemodialysis which evaluates attention and peritoneal dialysis presented a worst performance in the backward digit span which evaluates procedural memory. A study was conducted by Gamvrula K et al. ${ }^{[25]}$ They assessed the digit span test in CKD patients on HD and CAPD. Their findings were CAPD patients scored less in digit span test compared to HD patients.

Wang $\mathrm{C}$ et al. ${ }^{[26]}$ Stated that obesity affects cognition predominantly by affecting the organizations of brain, disproportion of leptin - insulin ratio, oxidative stress and cerebrovascular function, which result in the worsening of cognitive functions. In our case, inspite of having a normal $\mathrm{BMI}$ in the CKD patients in treatment group, we noticed cognitive impairment in them which clearly indicate that it was exclusively due to the disease process.

There is a neurocognitive deficit in patients on conservative management when compared to the patients on dialysis. The neurocognitive defects observed in CKD are poorly understood. The probable mechanisms causing cognitive impairment in CKD patients are metabolic neuronal injury, subclinical vascular disease were proposed to cause neuronal damage leading to neurocognitive dysfunction. ${ }^{[27]}$ Neuroimaging studies

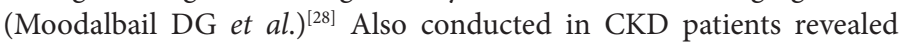
cortical atrophy, cerebral white matter lesion, cerebral density changes, signs of cerebral vascular disease and reduced hippocampal volume. All these factors might be contributing for altered neurocognitive function in CKD leading to cognitive decline. Kim et al. ${ }^{[29]}$ Found that white matter lesion is associated with cognitive impairment in CKD patients on CAPD. In hemodialysis patients, the dialysis process itself results in intravascular fluid loss and cause shifting of fluid which leads to cerebral edema that in turn causes diminished cerebral perfusion and cerebral ischemia leading to cognitive impairment.

\section{CONCLUSION}

\section{Based on the findings observed, we concluded that:}

Stage 5 CKD patients on conservative management has prolonged, TMT A and TMT B scores, LCT scores and increased LCT omissions and reduced FDS and BDS which indicates that cognitive functions are more affected in stage $5 \mathrm{CKD}$ patients on conservative management compared to $\mathrm{CKD}$ on $\mathrm{HD}$ and CAPD.

\section{ACKNOWLEDGEMENT}

We acknowledge the entire department of Physiology, Nephrology and Biochemistry for their support in completing my project.

\section{CONFLICT OF INTEREST}

The authors declare that there is no conflict of interest.

\section{ABBREVIATIONS}

CKD: Chronic kidney disease; HD: Hemodialysis; CAPD: Continuous ambulatory peritoneal dialysis; MMSE: Mini Mental State Examination; TMT: Trail making test; BDS: Backward digit span; FDS: Forward digit span; LCT: Letter cancellation test; eGFR: estimated glomerular filtration rate; WISC: Wechsler intelligence scale for children and adult; PASAT: Paced auditory serial addition test.

\section{REFERENCES}

1. Varma PP. Prevalence of chronic kidney disease in India - Where are we heading? Indian J Nephrol. 2015;25(3):133-5.

2. Wein AJ, Kavoussi LR, Novick AC, Partin AW, Peters CA. Campbell-Walsh Urology. Elsevier Health Sciences. 2011;5689.

3. Bello AK, Alrukhaimi M, Ashuntantang GE, Basnet S, Rotter RC, Douthat WG, et al. Complications of chronic kidney disease: current state, knowledge gaps and strategy for action. Kidney Int Suppl. 2017;7(2):122-9.

4. Thomas R, Kanso A, Sedor JR. Chronic kidney disease and its complications. Prim Care. 2008;35(2):329-44

5. Arnold R, IssarT, Krishnan AV, Pussell BA. Neurological complications in chronic kidney disease. JRSM Cardiovasc Dis. 2016;3:5.

6. Ryan JJ, Souheaver GT, DeWolfe AS. Intellectual deficit in chronic renal failure. A comparison with neurological and medical-psychiatric patients. J Nerv Ment Dis. 1980;168(12):763-7.

7. Souheaver GT, Ryan JJ, DeWolfe AS. Neuropsychological patterns in uremia. J Clin Psychol. 1982;38(3): 490-6.

8. Tsai CF, Wang SJ, Fuh JL. Moderate chronic kidney disease is associated with reduced cognitive performance in midlife women. Kidney Int. 2010;78(6):605-10

9. Gelder MG, Ibor JJL, Andreasen NC. New Oxford Textbook of Psychiatry. Oxford University Press. 2000;2131.

10. Sehgal AR, Grey SF, DeOreo PB, Whitehouse PJ. Prevalence, recognition and implications of mental impairment among hemodialysis patients. Am J Kidney Dis off J Natl Kidney Found. 1997;30(1):41-9.

11. Kurella M, Chertow GM, Fried LF, Cummings SR, Harris T, Simonsick E, et al. Chronic kidney disease and cognitive impairment in the elderly: the health aging and body composition study. J Am Soc Nephrol. 2005;16(7):2127-33.

12. Kurella TM, Muntner P, Wadley V, Cushman M, Zakai NA, Bradbury BD, et al. Albuminuria, kidney function and the incidence of cognitive impairment among adults in the United States. Am J Kidney Dis of J Natl Kidney Found. 2011;58(5):756-63.

13. Helmer C, Stengel B, Metzger M, Froissart M, Massy ZA, Tzourio C, et al Chronic kidney disease, cognitive decline and incident dementia: the 3C Study. Neurology. 2011;77(23):2043-51.

14. Condé SA, Fernandes N, Santos FR, Chouab A, Mota MMEP, Bastos MG Cognitive decline, depression and quality of life in patients at different stages of chronic kidney disease. Braz J Nephrol. 2010;32(3):242-8.

15. Stivelman JC. Benefits of anaemia treatment on cognitive function. Nephro Dial Transplant off Publ Eur Dial Transpl Assoc Eur Ren Assoc. 2000;15(Suppl 3):29-35.

16. Gaudino EA, Geisler MW, Squires NK. Construct validity in the Trail Making Test: 
what makes Part B harder?. J Clin Exp Neuropsychol. 1995;17(4):529-35.

17. Schneider SM, Malecki AK, Müller K, Schönfeld R, Girndt M, Mohr $P$, et al. Effect of a single dialysis session on cognitive function in CKD5D patients: a prospective clinical study. Nephrol Dial Transplant. 2015;30(9):1551-9.

18. Slinin Y, Paudel ML, Ishani A, Taylor BC, Yaffe K, Murray AM, et al. Kidney function and cognitive performance and decline in older men. J Am Geriatr Soc. 2008;56(11):2082-8.

19. Silverwood RJ, Richards M, Pierce M, Hardy R, Sattar N, Ferro C, et al. Cognitive and Kidney Function: Results from a British Birth Cohort Reaching Retirement Age. PLoS One. 2014;9(1):1-10.

20. Tsai CF, Wang SJ, Fuh JL. Moderate chronic kidney disease is associated with reduced cognitive performance in midlife women. Kidney Int. 2010;78(6):605-10.

21. Elias MF, Elias PK, Seliger SL, Narsipur SS, Dore GA, Robbins MA. Chronic kidney disease, creatinine and cognitive functioning. Nephrol Dial Transplant. 2009;24(8):2446-52.

22. Paraizo AMD, Almeida ALM, Pires LA, Abrita RSA, Crivellari MHT, Pereira SBD, et al. Montreal Cognitive Assessment (MoCA) screening mild cognitive impairment in patients with Chronic Kidney Disease (CKD) pre-dialysis. Braz J Nephrol. 2016;38(1):31-41.

23. Drew DA, Weiner DE, Tighiouart H, Duncan S, Gupta A, Scott T, et al. Cognitive Decline and its Risk Factors in Prevalent Hemodialysis Patients. Am J Kidney Dis off J Natl Kidney Found. 2017:69(6):780-7.
24. Dixit A, Thawani R, Goyal A, Vaney N. Psychomotor performance of medical students: effect of 24 h of sleep deprivation. Indian J Psychol Med. 2012;34(2):129.

25. Gamvrula K, Bozikas V, Holeva V, Griveas I, Sakelariou G, Karavatos A. Comparison of cognitive functioning between patients receiving hemodialysis versus peritoneal dialysis. Ann Gen Psychiatry. 2006;5(S1):S72.

26. Wang C, Chan JSY, Ren L, Yan JH. Obesity Reduces Cognitive and Motor Functions across the Lifespan. Neural Plast. 2016;1-3.

27. Tryc AB, Alwan G, Bokemeyer M, Goldbecker A, Hecker $H$, Haubitz $M$, et al. Cerebral metabolic alterations and cognitive dysfunction in chronic kidney disease. Nephrol Dial Transplant off Publ Eur Dial Transpl Assoc Eur Ren Assoc. 2011;26(8):2635-41.

28. Moodalbail DG, Reiser KA, Detre JA, Schultz RT, Herrington JD, Davatzikos C, et al. Systematic review of structural and functional neuroimaging findings in children and adults with CKD. Clin J Am Soc Nephrol CJASN. 2013;8(8):1429-48.

29. Kim CD, Lee HJ, Kim DJ, Kim BS, Shin SK, Do JY, et al. High prevalence of leukoaraiosis in cerebral magnetic resonance images of patients on peritoneal dialysis. Am J Kidney Dis off J Natl Kidney Found. 2007;50(1):98-107.

Cite this article: Renugasundari M, Velkumary S, Parameswaran S, Nanda N. Neurocognitive Assessment in Stage 5 Chronic Kidney Disease Patients on Various Treatment Modalities. Int J Clin Exp Physiol. 2019;6(1):18-23. 